International Journal of Biomedicine I June 2019 - Volume 9, Issue Suppl_1: Abstracts From the Second Russian International Conference "Cryo-electron microscopy 2019: achievements and prospects"

ORAL ABSTRACT PRESENTATIONS

SESSION TITLE: COMPLEX AND EMERGING TECHNIQUES IN STRUCTURAL BIOLOGY

DOI: 10.21103/IJBM.9.Suppl_1.OR22

\title{
Abstract OR-22: In vitro Cryo Electron Tomography study of protective Dps-DNA co-crystallization
}

Roman Kamyshinsky ${ }^{1,2}$, Yury Chesnokov ${ }^{1,2}$, Liubov Dadinova ${ }^{2}$, Andrey Mozhaev ${ }^{2,4}$, Anton Orekhov ${ }^{1,2}$, Eleonora Shtykova ${ }^{2,3}$, Alexander Vasiliev ${ }^{1,2}$

${ }^{1}$ National Research Center "Kurchatov Institute”, Moscow, Russia; ${ }^{2}$ Shubnikov Institute of Crystallography of Federal Scientific Research Centre "Crystallography and Photonics”, Russian Academy of Sciences, Moscow, Russia; ${ }^{3}$ Semenov Institute of Chemical Physics of Russian Academy of

Sciences, Moscow, Russia; ${ }^{4}$ Shemyakin-Ovchinnikov Institute of bioorganic chemistry of Russian Academy of Sciences, Moscow, Russia

Background: It was shown that when exposed to extreme environment Dps-DNA cocrystallization takes place in Escherichia coli as a protective mechanism. However, to this date most of the proposed models of co-crystals were not backed up with any direct methods. Recent developments in Cryo Electron Tomography allow to visualize co-crystals with high spacial resolution in nearly native state.

Methods: The investigations were carried out in a Titan Krios 60-300 TEM/STEM (FEI, USA) CryoEM, equipped with direct electron detector Falcon II (FEI, USA) and Cs image corrector (CEOS, Germany), at accelerating voltage of $300 \mathrm{kV}$. Tilt series were collected automatically using FEI Tomography software in low-dose mode which allowed to minimize the radiation damage. Subtomogram averaging was performed in Relion2 software package. Data processing and 3D reconstruction were carried out using computing resources of the Federal Collective Usage Center Complex for Simulation and Data Processing for Mega-Science Facilities at NRC "Kurchatov Institute".

Results: In this study, different types of Dps-DNA co-crystals were observed in vitro. Structural characteristics of the co-crystals were revealed for the first time by Cryo Electron Tomography. Subtomogram averaging allowed the direct visualization of Dps and DNA molecules in co-crystals. It was shown that the first type of co-crystals exhibits lamellar morphology and is formed by Dps layers alternating with DNA strands. The co-crystals adopt central symmetric triclinic crystal structure. The second type adopts body-centered cubic crystal lattice with mutually perpendicular DNA strands packed between Dps molecules. We assume that co-crystals' structure could be dependent on the parameters of the utilized Dps and DNA solutions. 
Conclusion: In this study, we established structural characteristics of different types of Dps-DNA co-crystals. The obtained results are a vital step towards understanding the mechanism of protective DpsDNA co-crystallization process.

Key Words: biocrystallization $\bullet$ Dps $\bullet$ DNA $\bullet$ nanocrystals

Sources of Funding: This work was supported by the Russian Science Foundation (Project № 18-7410071).

International Journal of Biomedicine. 2019;9 Suppl 1: S15. doi: 10.21103/IJBM.9.Suppl_1.OR22

(C)2019 International Medical Research and Development Corporation 